\title{
Strengthening health decision-making at the territorial level: operational support for spatial multi-criteria evaluation
}

\author{
Annelise TRAN - François ROGER
}

Animal diseases and zoonoses, infectious diseases that can be transmitted between animals and humans, are a global socio-economic and public health problem. Ensuring the surveillance and control of these diseases implies identifying high risk areas. Maps of these areas can be produced using spatial multi-criteria evaluation, a recent method that is rigorous and quick to implement. Animal health and veterinary public health policymakers are still relatively unfamiliar with this method, but it is suited to regions with little access to reliable epidemiological data, such as low-income countries in the South or disease-free areas. The maps are built with local experts in the regions concerned. They present risk indices combining geographical and environmental data, expert opinions and information from existing studies and surveys. These maps can then be used to better target disease surveillance and control.
$\mathrm{T}$ better target the surveillance and control of animal or zoonotic diseases potentially emerging or present in a territory, the first step is to identify high risk areas. For a given disease and territory, the risks are the introduction of the pathogen, its amplification (its local transmission, creating a primary outbreak), its spread (its transportation from a primary outbreak to one or more secondary outbreaks], its maintenance, and the socio-economic consequences associated with its occurrence.

Risk-based surveillance has two operational advantages: it helps to streamline the use of human and financial resources, which are very limited in low-income countries in the South, and is well suited to the detection of new diseases in both the South and the North. However, this type of surveillance is based on qualitative, empirical methods that are difficult to assess, or on more conventional mapping methods requiring large health datasets. But such datasets are often unavailable in the countries of the South and, in areas free from a potentially emerging disease, health data for the construction of models or the validation of maps is of course non-existent. Consequently, the environmental risk factors of a disease are not always identified or quantified, making it impossible to evaluate the risks of amplification and spread, and especially of endemisation.
It is therefore essential to develop methods that do not require large health datasets and which take account of all factors for the risk studied. This is the case of the spatial multi-criteria evaluation method (or spatial MCE), which is knowledge-based, and is still rarely used by animal health and veterinary public health policymakers (see box p. 2).

\section{Spatial multi-criteria evaluation,}

a solution for regions lacking epidemiological and health data

Examples of the application of spatial multi-criteria evaluation to zoonoses, such as avian influenza in Southeast Asia and Rift Valley fever in East Africa, show that this approach is robust and relevant (see p. 3).

In contexts with little data from surveys or surveillance systems, this method is an interesting solution that is relatively simple to implement. It can be adapted to different geographical scales (international, national, local), enabling appropriate decisions to be made at each level. It integrates expert opinions to identify the environmental factors and husbandry practices that foster the introduction or transmission of diseases. Based on this knowledge, it produces maps of high risk areas that health actors in a given 


\section{Stages of spatial multi-criteria evaluation}

\section{The six key stages}

1- Defining the risk and the territory of interest. The risk to be mapped (risk of introduction, amplification, spread, maintenance, etc.) and the targeted territory, with the basic geographical unit of calculation (municipality, district, region, $1 \mathrm{~km}^{2}$ grid, etc.).

2- Identifying the factors, or criteria, that play a known or assumed role in this risk: environmental factors, animal densities, husbandry practices, etc. Analysis of literature reviews and discussions with local experts [epidemiologists, ecologists, entomologists, specialists in human or animal health, etc.].

3- Weighting these factors based on expert opinions. Assigning a score, or weight, of between 0 and 1 according to their relative importance. If close to 0 , the factor contributes little to the risk; but if close to 1 , the factor is decisive. Methods are used to translate the qualitative opinions of experts into numerical values.

4- Collecting geographical data corresponding to the factors identified. Depending on the calculation unit, this data is collected on the ground or obtained from national institutes or from geographical databases on the Internet.

5- Creating spatial, standardised suitability indices. For each basic geographical unit, the geographical data is transformed to give each criterion a numerical value reflecting the risk according to the criterion studied: from 0 (very low risk), to 1 (high risk). This transformation reflects the relationship between the criterion and the risk studied, a relationship that may take different forms [increasing, decreasing, with an optimum, linear or non-linear] and must translate qualitative expert opinions.

6- Combining spatial suitability indices to produce a risk map. For each basic geographical unit, a synthetic risk index is obtained, for example, by calculating the sum of the suitability indices calculated in stage 5 and weighted. The result is a map presenting each geographical unit with its risk value. A geographical unit will thus have a higher risk value [close to 1 ) if the indices calculated in stage 5 have a high value.

\section{Depending on the conditions, two additional stages}

7- Analysing uncertainty and sensitivity associated with the risk map. This consists in varying the weight allocated to each factor: with different weights, do we obtain the same map? When experts give different opinions, what is the impact on the variability of the result? An uncertainty map can then be associated with the risk map.

8- Validating the risk map by comparing the values of the risk index calculated for the different geographical units with epidemiological data for the disease in question, where available. This stage helps to assess the relevance of the map before using it for surveillance. In example 1 on avian influenza (see p. 3), the method was applied and validated in Thailand; validation showed its high predictive value and justified its use in Cambodia.

\section{And the essential stage of explanation to end users}

It is crucial to communicate to end users all information concerning map production: choice and weighting of risk factors, sources of geographical data, uncertainty map, etc. This stage is often missing in the application of risk analyses. territory can use to implement surveillance actions. These maps summarise knowledge of a disease at a given time: they can also be updated according to new knowledge acquired, such as an additional risk factor or changes to the relative importance of different factors.

Spatial multi-criteria evaluation has the advantage of mapping a health risk without using data on the occurrence of the health problem targeted. It is thus complementary to other models used in epidemiology such as statistical models of the distribution of disease occurrence and mechanistic models of spread. However, it could be considered as subjective, since it is built on the knowledge and expert opinions of actors concerned. The reliability of the method is therefore based first on the quality of this expertise and, second, on the quality of data reflecting geographical variations in the risk factors taken into account.

Where health data is available, it can be used to assess the quality of the risk map: in the examples presented, in Southeast Asia and East Africa, this validation has shown in particular the robustness of the method in terms of mapping the risks of disease transmission.

\section{Training practitioners in the use of spatial multi-criteria evaluation}

Spatial multi-criteria evaluation requires a good grasp of IT tools, such as geographical information systems (GIS), which are essential when using geographical data and producing knowledge-based risk maps. To ensure health actors adopt this method, training on GIS tools and risk mapping methods must be organised. In addition, the adaptation of easy-to-use GIS tools would foster wider use: for example, the Institut Pasteur de Madagascar is working on an extension to the open-source geographical information system application QGIS, which will enable the method to be implemented.

Another technical constraint is access to geographical and environmental databases characterising the risk studied, such as animal distribution maps, types of vegetation, presence of water, etc. This data is not always available, but free data can very often be found on the internet, enabling an initial estimation. Geographical information portals provide this kind of environmental database. This is the case of the European E3 Geoportal (European Environment and Epidemiology Network, European Centre for Disease Prevention and Control] and the AWARE portal (CIRAD's Agricultural Web Atlas for Research). Again, training must be organised for users to enable them to access, download and integrate this data into a geographical information system. 


\section{Example 1.}

\section{Mapping of areas at risk of avian influenza transmission in poultry in Thailand and Cambodia}

Stage 1, territory of interest. The multi-criteria evaluation method was applied and validated in Thailand then deployed in Cambodia. This low-income country has little epidemiological data. The maps obtained identify high risk areas for priority surveillance.

Stage 2, risk factors. Free-ranging ducks, farm chickens and ducks, roads and markets, water and rice paddies, human population, etc.

Stage 3, factors weighted by experts. Depending on the country, the factors have different weights, but free-ranging ducks are highest.

Stage 4, geographical data. Density of free-ranging ducks, density of farm chickens and ducks, density of road network, proximity of markets, density of hydrographic network, surface area of rice paddies.

Stage 5, spatial, standardised suitability indices, varying between 0 and 1 for each of the factors.

Stage 6, risk map.

From green

[low risk areas], to red [high risk areas].

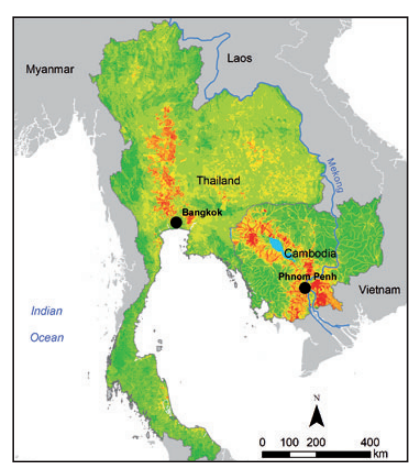

Stage 7, map uncertainty. From green-blue (low uncertainty), through pale yellow [medium uncertainty), to brown, higher uncertainty.

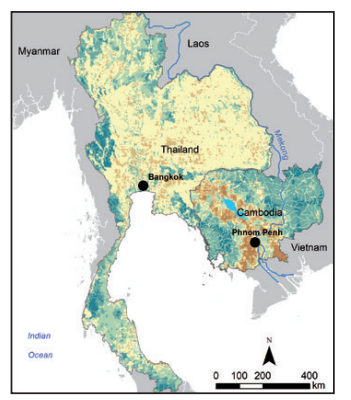

\section{Example 2.}

\section{Mapping of zones potentially suitable for the amplification and spread of Rift Valley fever virus in East Africa [Ethiopia, Uganda, Kenya, Tanzania]}

The risk factors identified are the same for both processes, but the weight of each factor is different: during amplification, vector mosquitoes are predominant in the transmission of the virus, whereas trade in ruminants is the primary factor in the spread of the virus.

The final risk map, combining amplification and spread, was compared with outbreaks declared in Kenya and Tanzania: this validation has shown the robustness of the approach in establishing risk maps in disease-free neighbouring countries.

\section{Risk map:}

(a) amplification risk map - [b] spread risk map. From green [low], through yellow (medium], to dark red (very high).

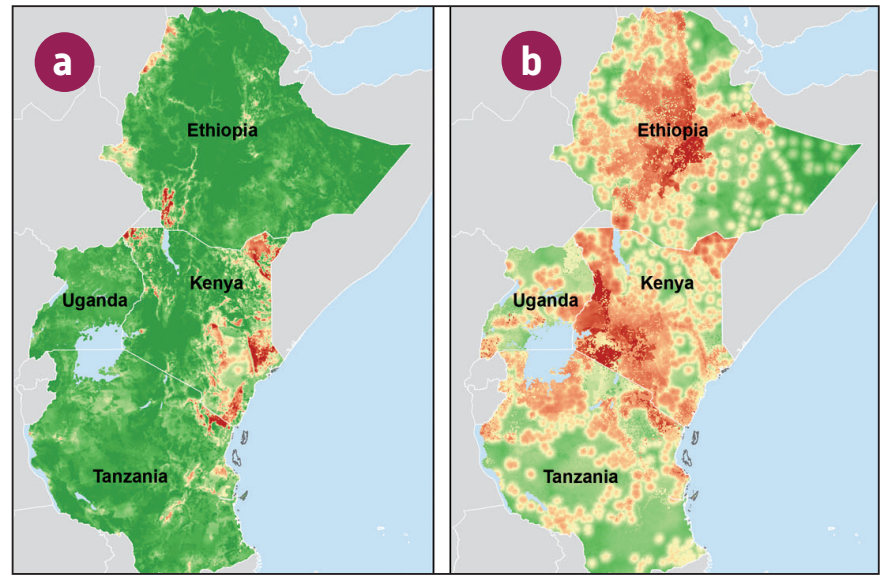

The maps are taken from the open access article: Tran A. et al. [2016] PLoS Neglected Tropical Diseases 10[9]: e0004999. https://doi.org/10.1371/journal.pntd.0004999

The maps are taken from the open access article: Paul M. C. et al. [2016] Scientific Reports 6, 31096. https://doi.org/10.1038/srep31096

\section{Outlook: fine-tuning methods}

\section{and using them in a One Health context at the territorial level}

The effectiveness of the spatial multi-criteria evaluation method presented here paves the way for methodological developments useful to health risk management in a given territory.

Multi-criteria decision analysis (MCDA) methods could also be developed for decision support based on the clarification of different scenarios. For a given disease, several maps are built according to different user hypotheses. These maps are used to discuss knowledge of disease transmission, such as the role of a wild reservoir or of trade. They define areas where the probability of detecting a disease is high, and they also take into account the risk of a high spread rate, or a high risk of maintenance, or an area that will suffer the greatest socio-economic impacts.

Spatial multi-criteria evaluation could be combined with other methods used in epidemiology, such as social network analysis models, in order to more accurately estimate risks.

It could also be used in conjunction with participatory mapping, implemented at a very local level. The actors (livestock farmers, medical staff, veterinarians, etc.) draw a map directly, which could be connected to the risk maps established for larger areas.

Non-spatial multi-criteria evaluations could also be used to support decision-making for disease control or to prioritise 
choices, such as defining priority diseases. This is the case of the tool for human diseases at the European Centre for Disease Prevention and Control.

All of these methods combine risk factors from different sectors and disciplines and can thus help to better integrate knowledge into health actions and policy. In particular, they can contribute to implementing on the ground systemic health approaches at different levels - One Health,
EcoHealth, Planetary Health - at the crossroads between human, animal and environmental health.

In the longer term, the development of artificial intelligence methods in the field of health could lead to the definition of algorithms to capture and organise the data available, including textual data. These algorithms could supplement the aspects under human supervision (experts] with a view to proposing predictive risk maps.
Perspective $n^{\circ} 46$ is the result of research conducted by the authors and their partners in Europe, with a project financed by FAO for East Africa through the FAO One Health Project OSRO/GLO/104/IRE, www.fao. org/ag/againfo/programmes/en/empres/news_220213. html\#note1], and in Asia through the REVASIA programme (Research for Evaluation of Animal Health Surveillance and Control in Southeast Asia, http://revasia.cirad.fr/], as part of the Platform for partnership in research and training GREASE [Management of emerging risks in Southeast Asia, www.grease-network.org/].

This research has also given rise to the following publications:

Paul M. C., Goutard F. L., Roulleau F., Holl D., Thanapongtharm W., Roger R. L., Tran A., 2016. Quantitative assessment of a spatial multicriteria model for highly pathogenic avian influenza H5N1 in Thailand, and application in Cambodia. Nature Scientific Reports 6:31096. https://doi.org/10.1038/srep31096.

Tran A., Trevennec C., Lutwama J., Sserugga J., Gély M., Pittiglio C., Pinto J., Chevalier V., 2016. Development and Assessment of a Geographic Knowledge-Based Model for Mapping Suitable Areas for Rift Valley Fever Transmission in Eastern Africa. PLoS Neglected Tropical Diseases 10[9]: e0004999. https://doi.org/10.1371/journal.pntd.0004999.

Tran A., Ippoliti C., Balenghien T., Conte A., Gely M., Calistri P., Goffredo M., Baldet T., Chevalier V., 2013. A geographical information system-based multicriteria evaluation to map areas at risk for rift valley fever vector-borne transmission in Italy. Transboundary and Emerging Diseases 60 (suppl. 2): 14-23. https://doi.org/10.1111/tbed.12156.

\section{A few links}

Aenishaenslin C., Hongoh V., Djibrilla Cissé H., Gatewood Hoen A., Samoura K., Michel P., Waaub J.-P., Bélanger D., 2013. Multi-criteria decision analysis as an innovative approach to managing zoonoses: results from a study on Lyme disease in Canada. BMC Public Health 13:897. https://doi.org/10.1186/1471-2458-13-897.

AWARE, Agricultural Web Atlas for research on agriculture, environment and tropical agronomy [CIRAD]. http://aware.cirad.fr/.

ECDC (European Centre for Disease Prevention and Control] tool for the prioritisation of infectious disease threats https://ecdc.europa.eu/en/publications-data/ecdc-toolprioritisation-infectious-disease-threats.

European Environment and Epidemiology [E3] Network. E3 Geoportal. https://e3geoportal.ecdc.europa.eu.

Institut Pasteur de Madagascar. EPI-GISVEC Project, GIS and VEctor Control program to identify priority areas for insecticide residual spraying. www.pasteur.mg/projets/gis-and-vector-control-program-toidentify-priority-areas-for-insecticide-residual-spraying/.

QGIS, open-source geographical information system application. www.qgis.org/.

This article is provi-
ded under the terms
of the Creative Com-
decision-making at the territorial level: operational support for spatial multi-criteria evaluation. CIRAD, Montpellier, Perspective 46. https://doi.org/10.19182/agritrop/00022.

\section{A few words about...}

Annelise Tran is a researcher in geomatics at CIRAD in the TETIS Joint Research Unit (Land, Environment, Remote Sensing and Spatial Information, https://tetis.teledetection.fr/index.php/fr/] and an associate researcher in the ASTRE Joint Research Unit. She is based in Réunion. Her activities focus on the development of remote sensing and spatial modelling methods with applications in the field of health.

\section{annelise.tran@cirad.fr}

François Roger is a veterinarian and epidemiologist at CIRAD where he is co-director of the ASTRE Joint Research Unit (Animals, Health, Territories, Risks and Ecosystems, http://umr-astre.cirad.fr/]. After a number of years working in sub-Saharan Africa, Europe and Asia, he is now based in Réunion, where he coordinates several crosscutting, multi-site studies in the field of health.

\section{francois.roger@cirad.fr}

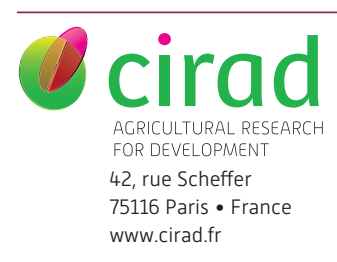

Publication Director: Michel Eddi,

CIRAD President Managing Director

Editors: Patrick Caron, Office of the Director General

in charge of Research and Strategy

Cécile Fovet-Rabot,

Scientific and Technical Information Service

Translation: Anna Kiff

Graphic design: Delphine Lavastre-Guard,

Communication Service

Distribution: Christiane Jacquet, Communication Service

www.cirad.fr/en/publications-ressources/edition/perspective-policy-brief

perspactive ISSN-L 2275-9190 - Email: perspective@cirad.fr

\section{a. \\ M U U S E}

MONTPELIIER UNIVERSTIÉD'EXCELLENCE http://muse.edu.umontpellier.fr/ 